\title{
Optimized Partial Decoding in Uplink Network MIMO under Constraint Backhaul
}

\author{
Shipeng Wang, Li Chen and Guo Wei \\ Wireless Information Network Laboratory, \\ University of Science and Technology of China, Hefei, China \\ Email: \{shpwang, clbyx\}@mail.ustc.edu.cn, wei@ustc.edu.cn
}

\begin{abstract}
This paper considers the model that each base station (BS) is connected to a central processor (CP) via a noiseless and rate-limited backhaul link. The theoretical capacity of this model is still left open. In our work, we use data splitting to explore the achievable rate region under given capacities of backhaul links. The performance of the data splitting is analyzed, and the optimal power allocation can be derived by solving the Karush-Kuhn-Tucker (KKT) conditions. An optimization method is also used to obtain the boundary of achievable rate region instead of complex exhaustive search. In the numerical simulations, the boundary of achievable rate region is presented. The result reveals that the rate region of the proposed scheme is superior to that of joint decoding scheme and Interference Channel (IC). The sum rate of our proposed scheme is also larger than that of the other two schemes.
\end{abstract}

\section{INTRODUCTION}

In modern cellular systems, network capacity is mainly limited by inter-cell interference. The limitation is particularly severe near the cell edge where the quality of service deteriorates sharply due to strong interference from the nearby cells. To address this issue, joint multicell processing (MCP) or cooperation of multi-point (CoMP) is adopted as a superior approach to eliminate inter-cell interference [1][2]. In the cooperation among BSs, joint decoding in the uplink and joint transmission in the downlink are employed to overcome the interference effect. Theoretically, the interference can be eliminated entirely when BSs share all the data and perfect channel state information (CSI) with each other. Excellent decoding can be performed, though, at the cost of extra backhaul links via which Base Stations exchange the BSs' signals and the CSI.

The inchoate concept of network multi-input multi-output (MIMO) is given rise to in [3]. The capacity region can be easily obtained with infinite backhaul link capacity, because the downlink scenario can be degraded to a Broadcast Channel (BC) and the uplink network MIMO can be degraded to a Multiple Access Channel (MAC). But in practical uplink scenario, we must take the effect of finite capacity in the backhaul into consideration. However, the theoretical capacity of finite backhaul links in uplink model is still an open problem. This motivates us to explore achievable rate region of uplink network MIMO model under constraint backhaul.

Researchers have studied uplink network MIMO with finite-capacity backhaul in some of the latest research proposals. In these researches, there are two major kinds of situations. One is that the BSs are connected with each other for cooperation. The other one is that the BSs are connected to a $\mathrm{CP}$ for cooperation. In the former situation, distributed interference substraction scheme is applied to acquire an achievable data rate in [4]. Then in [5], a comprehensive analysis of rate region under different cooperative schemes provides new insight into the value of network MIMO. Furthermore, the common message (which both BSs decoded) and private message (which only supporting BS decoded) are also utilized to explore the rate region. However, about the second situation, an achievable rate from user to the $\mathrm{CP}$ is given based on a single-user scenario in [6], and whether the BSs are capable of decoding or not is considered. In [7], a multi-user system, in which Wyner-ZiV compress-and-forward scheme is deployed on the BSs and successive interference cancellation (SIC) is applied at the CP, is studied. The author derives a suboptimal achievable rate region that is easily computable. But the optimal achievable rate region of uplink network MIMO where the BSs are connected to the CP has not been explored. What's more, partial decoding, which means that the BSs are able to decode partial message from users, is not considered in the latter situation.

In this paper, we focus on the uplink network MIMO scenario in which each BS is connected to a CP via a noiseless and rate-limited backhaul link. We divide the data stream of each user into two parts, which are independent decoding data and joint decoding data respectively. The independent decoding data are decoded in the local BSs, while the joint decoding data are decoded at the $\mathrm{CP}$. We attempt to explore the achievable rate region of this model. Under the given constraints of backhaul links, we also want to answer the following two problems:

- How useful are partial decoding in the BSs and joint decoding in the $\mathrm{CP}$ ? We compare the achievable rate region among IC, joint decoding scheme and proposed scheme.

- If only parts of the message are decoded in supporting BSs, i.e. not all messages can be decoded by BSs, what is the best rate we can achieve.

In our model, there are two users and two base stations. For each user equipment (UE), we split data streams into two parts which are implemented by superposition coding. Intuitively, the approach can reduce the burden of $\mathrm{CP}$ decoding and make full use of the finite backhaul link. This is because the BSs help decoding some data, so that less signal processing occurs in the CP. Further, if the capacities of backhauls are too small, the indexes of the message in the codebooks need to be transmitted to the $\mathrm{CP}$ to increase data rate. However, if the backhaul is 
sufficiently large, the quantization noise power will be much smaller, and the joint decoding can obtain better data rate. In our work, the rate region is defined as the operation of convex hull to achievable rate pair under all the power allocations. Instead of the exhaustive method, we propose an optimization method to obtain the boundary of the achievable rate region with low complexity.

The paper is organized as follows. In Section II, we briefly introduce the model of uplink network MIMO system and transmission scheme. In Section III, the rate region of the proposed scheme of network MIMO system is analyzed. An optimization method to acquire the boundary of achievable rate region is also proposed in this section. The numerical simulation is provided in Section $\mathrm{V}$, and finally, the conclusions are drawn in Section VI.

\section{System Model And TRAnSmission Scheme}

In this section, we consider an uplink model of multi-cell network, as shown in Fig.1. In this paper, each receiver is equipped with $N_{r}$ antennas, but each transmitter has only one antenna. The BSs are connected to the $\mathrm{CP}$ or backbone network via a noiseless backhaul link with capacity $C_{i}, i=1,2$. The coefficient $H_{i j} \in \mathbb{C}^{N_{r} \times 1}$ is the $N_{r}$-dimensional complex vector corresponding to the channel between user $j$ and $\mathrm{BS} i$. Since we consider the uplink scenario, the CSI can be acquired by channel estimation on the BSs. In addition, perfect CSI is assumed in the BS and CP because we mainly concern the performance of partial decoding. Thus the signals received at BS $i$ will be given by

$$
Y_{i}=\sum_{j=1}^{2} H_{i j} X_{j}+Z_{i}, i=1,2,
$$

where $X_{i} \in \mathbb{C}$ denotes user $i$ 's transmit data, and $Z_{i} \in$ $\operatorname{e\mathcal {N}}\left(0, \sigma^{2} I\right)$ is the receiver noise. $X_{i}$ is subject to power constraint $P_{i}$ as

$$
\mathbb{E}\left\|X_{i}\right\|^{2} \leq P_{i}, i=1,2 .
$$

In IC scenario, the BSs decode the user data under the interference from each other as a result of no cooperation between the BSs. While in network MIMO situation, the BSs will compress their received signals and forward them to the $\mathrm{CP}$, where the user data are jointly decoded by the compressed signal $\widehat{Y}_{i}, i=1,2$. In an attempt to bridge the IC scenario (when the data are decoded in the BSs) and the network MIMO situation (where they are not), we propose to split the user data across two parts of messages:

- Independent decoding data, which is decoded by the corresponding BS.

- Joint decoding data which is jointly decoded in the CP.

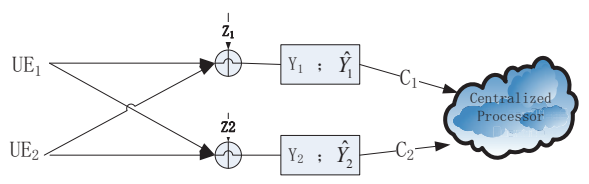

Fig. 1. Uplink transmission model with a central processor.
Therefore, the rate $r_{i}$ of user $i$ will be split to $r_{i p}$ and $r_{i c}$, which respectively refer to the rate of independent decoding data and joint decoding data for that user, i.e.

$$
r_{i}=r_{i p}+r_{i c}, i=1,2 \text {. }
$$

- By forcing $r_{i}=r_{i p}, r_{i c}=0$, our transmission scheme can be degraded to a "X" interference channel.

- By forcing $r_{i}=r_{i c}, r_{i p}=0$, our transmission scheme can be degraded to a network MIMO scenario.

The general situation is that both $r_{i p}$ and $r_{i c}$ are not zero. In our proposed scheme, decoding in the BSs can relieve burden of the $\mathrm{CP}$, while decoding in the $\mathrm{CP}$ can use jointly decoding to remove the interference. Intuitively, there is a tradeoff problem between the two particular rates.

We assume that the users' data can be decoded in the supporting $\mathrm{BS}$ or the $\mathrm{CP}$, but must be eventually transmitted to the CP. Under the assumption, we introduce several fundamental inequalities imposed by the constraint backhaul. For the independent decoding data, the constraint of the rate $r_{i p}$ applies:

$$
r_{i p} \leq C_{i}, i=1,2 .
$$

Furthermore, the sum rate $r_{1}+r_{2}$ cannot exceed the total backhaul capacity

$$
r_{1}+r_{2} \leq C_{1}+C_{2}
$$

\section{ACHIEVAble RATE REgION}

In this section, we will specify how our transmission scheme is implemented by superposing coding and conventional decoding. Then an achievable rate region is defined under an specific decoding scheme in the $\mathrm{CP}$. We also introduce an optimized method to obtain the boundary of such an achievable rate region.

\section{A. Superposed Code}

The overall channel can be regarded as the superposition of the two particular situations, thus we write the data $X_{i}$ of user $i$ as superposition of two data, $x_{i p}, x_{i c}, i=1,2$, i.e.

$$
X_{i}=\sqrt{\rho_{i p}} x_{i p}+\sqrt{\rho_{i c}} x_{i c}, i=1,2,
$$

where both $x_{i p}$ and $x_{i c}$ are independent $\mathcal{C N}(0,1)$ random variables, and $\rho_{i p}, \rho_{i c}$ are the power divided to each data. It's worth mentioning that $x_{i p}$ denote the independent decoding data which are decoded at the BSs, and the $x_{i c}$ denote the joint decoding data which is decoded in the $\mathrm{CP}$. According to (2), we have

$$
\begin{aligned}
\mathbb{E}\left\|X_{i}\right\|^{2} & =\mathbb{E}\left\|\sqrt{\rho_{i p}} x_{i p}+\sqrt{\rho_{i c}} x_{i c}\right\|^{2} \\
& =\rho_{i p}+\rho_{i c} \\
& \leq P_{i}, \quad i=1,2,
\end{aligned}
$$

which is the constraint of the power allocation in the transmitter. We are also able to obtain the two particular cases by this power allocation. For example, by forcing $\rho_{i p}=0, \rho_{i c} \neq 0$, there is the IC situation, while the network MIMO scenario is obtained by forcing $\rho_{i p} \neq 0, \rho_{i c}=0$. 
After each BS receives the signals $Y_{i}$, it will decode the data of user $i$ with the interference of user $\bar{i}$, data $X_{\bar{i}}$ and its own data $x_{i c}$. In what follows, $\bar{i}=\bmod (i, 2)+1, i=1,2$, denotes the other user or BSs based on the context. Thus, the rate constraint of user data between the user and the BSs can be given as

$$
\begin{aligned}
& r_{i p} \leq \log _{2}\left(1+\frac{\rho_{i p} H_{i i}^{H} H_{i i}}{\rho_{i c} H_{i i}^{H} H_{i i}+\left(\rho_{\bar{i} p}+\rho_{\overline{i c}}\right) H_{i \bar{i}}^{H} H_{i \bar{i}}+\sigma^{2}}\right), \\
& i=1,2 \text {. }
\end{aligned}
$$

Because all the data of users should be delivered to the $\mathrm{CP}$, the rate of data decoded in the BSs can not exceed the backhaul capacity (i.e. the max-flow-min-cut theory). Hence, the rate $r_{i p}$ constraint between the users and the $\mathrm{CP}$ is shown on the next page header. After BSs have decoded this part of data, they will subtract this signals and compress the left signals, then forward it to the CP. The left signals $\bar{Y}_{i}$ is given as

$$
\begin{aligned}
\bar{Y}_{i} & =Y_{i}-H_{i i} x_{i p} \\
& =H_{i i} x_{i c}+H_{i \bar{i}} X_{\bar{i}}+Z_{i}, i=1,2 .
\end{aligned}
$$

The compressed scheme will be introduced in detail in next subsection.

\section{B. Compressed-and-Forwarding Scheme}

Owing to the finite backhaul, the BSs can not forward the signals to the $\mathrm{CP}$ without losses. Based on the rate-distortion theory, we need to compress the signal by the model of test channel [8] and the model of bussgang [9][10],

$$
\widehat{Y}_{i}=a_{i} \bar{Y}_{i}+q_{i}, \text { for } i=1,2,
$$

where $q_{i}$ is Gaussian quantized noise and $a_{i}$ is real-valued. Because the transmit signal and the quantization noise are both gaussian random variables, the Lemma 1 can be stated as follows, which helps us derive the coefficient and the quantized noise in (11).

Lemma 1: The rate-distortion function of signal received by $\mathrm{BSs}$ is given as:

$$
R\left(D_{i}\right)= \begin{cases}\log \left(\frac{\left\|\bar{Y}_{i}\right\|^{2}}{D_{i}}\right) & 0 \leq D_{i} \leq\left\|\bar{Y}_{i}\right\|^{2} \\ 0 & D_{i} \geq\left\|\bar{Y}_{i}\right\|^{2}\end{cases}
$$

where

$$
\begin{array}{r}
\left\|\bar{Y}_{i}\right\|^{2}=\bar{Y}_{i}^{H} \bar{Y}_{i} \\
E\left(\widehat{Y}_{i}-\bar{Y}_{i}\right) \leq D_{i} .
\end{array}
$$

Thus the constraint of backhaul link is given as

$$
R\left(D_{i}\right) \leq C_{i}-r_{i p}
$$

Proof: Both the data and the quantization noises are gaussian and independent, so the signals received by BS are gaussian. Thus according to rate-distortion theory in [8], the function can be given as (12). For the full use of the backhaul, it usually sets (15) to $R\left(D_{i}\right)=C_{i}-r_{i p}$. In this case, the distortion is minimized. In what follows, we will use the minimum distortion to obtain the best performance.

Based on the lemma above, we obtain the following theorem.
Theorem 1: When the capacity of backhaul is fully used, the coefficient and quantized noise in (11) are given as:

$$
\left\{\begin{array}{l}
a_{i}=1-\frac{D_{i}}{\left\|Y_{i}\right\|^{2}} \\
q_{i} \sim \mathcal{N}\left(0, D_{i}\left(1-\frac{D_{i}}{\left\|Y_{i}\right\|^{2}}\right)\right)
\end{array}\right.
$$

Proof: This theorem is derived based on the test channel shown in Fig.2. In this setting, $\bar{Y}_{i}$ and $\widehat{Y}_{i}$ are respectively the original gaussian signal and quantized signal. $q_{i}$ denotes the quantized noise. $\left\|\bar{Y}_{i}\right\|^{2},\left\|\widehat{Y}_{i}\right\|^{2}$ and $Q_{i}$ are their covariances respectively. Thus the mutual information is given as $I\left(\bar{Y}_{i} ; \widehat{Y}_{i}\right)=\log \frac{\left\|\widehat{Y}_{i}\right\|^{2}}{Q_{i}}$. Based on the equation (11), the covariance of quantized signal is

$$
\begin{aligned}
\left\|\widehat{Y}_{i}\right\|^{2} & =a_{i}^{2}\left\|\bar{Y}_{i}\right\|^{2}+Q_{i} \\
& =\left(1-\frac{D_{i}}{\left\|\bar{Y}_{i}\right\|^{2}}\right)^{2}\left\|\bar{Y}_{i}\right\|^{2}+D_{i}\left(1-\frac{D_{i}}{\left\|\bar{Y}_{i}\right\|^{2}}\right) \\
& =\left\|\bar{Y}_{i}\right\|^{2}-D_{i} .
\end{aligned}
$$

Thus the mutual information $I\left(\bar{Y}_{i} ; \widehat{Y}_{i}\right)$ is expressed as

$$
\begin{aligned}
I\left(\bar{Y}_{i} ; \widehat{Y}_{i}\right) & =\log \frac{\left\|\bar{Y}_{i}\right\|^{2}-D_{i}}{D_{i}\left(1-\frac{D_{i}}{\left\|\bar{Y}_{i}\right\|^{2}}\right)} \\
& =\log \frac{\left\|\bar{Y}_{i}\right\|^{2}}{D_{i}},
\end{aligned}
$$

which satisfies the result of Lemma 1. Therefore, the theorem 1 can achieve the lower bound of the rate-distortion function.

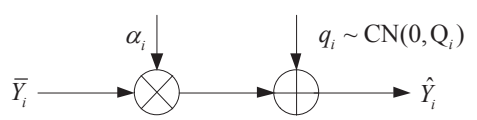

Fig. 2. The joint distribution of gaussian source in quantized model.

\section{Decoding Scheme in the $C P$}

When the $\mathrm{CP}$ receives the compressed signals, it will decode the users' data. Joint decoding scheme, which can eliminate interference to raise the data rate, will be utilized. As shown in [7], the performance of SIC scheme is already approximately optimal. Therefore, we assume the SIC scheme is used to decoded in the CP. Furthermore, the order of decoding is fixed as that the data of user 1 is firstly decoded, then the user 2 data is decoded without the interference from user 1 .

The following proposition specifies a rate region corresponding to the over-the-air channel.

Proposition 1: Given backhaul capacity and power allocation, the rate region is achievable on the over-the-air segment:

$$
\begin{aligned}
& r_{i p} \leq \min \left\{C_{i}, \log _{2}\left(1+\frac{\rho_{i p} H_{i i}^{H} H_{i i}}{\Phi_{i p}}\right)\right\}, i=1,2 \\
& r_{1 c} \leq \log _{2}\left(1+\frac{a_{1}^{2} \rho_{1 c} H_{11}^{H} H_{11}}{a_{1}^{2} \rho_{2 c} H_{12}^{H} H_{12}+a_{1}^{2} \sigma^{2}+Q_{1}}\right) \\
& r_{2 c} \leq \log _{2}\left(1+\frac{a_{2}^{2} \rho_{2 c} H_{22}^{H} H_{22}}{a_{2}^{2} \sigma^{2}+Q_{2}}\right)
\end{aligned}
$$




$$
r_{i p} \leq \min \left\{C_{i}, \log _{2}\left(1+\frac{\rho_{i p} H_{i i}^{H} H_{i i}}{\rho_{i c} H_{i i}^{H} H_{i i}+\left(\rho_{\bar{i} p}+\rho_{\overline{i c}}\right) H_{i \bar{i}}^{H} H_{i \bar{i}}+\sigma^{2}}\right)\right\}, i=1,2 .
$$

where

$$
\Phi_{i p}=\rho_{i c} H_{i i}^{H} H_{i i}+\left(\rho_{\bar{i} p}+\rho_{\overline{i c}}\right) H_{i \bar{i}}^{H} H_{i \bar{i}}+\sigma^{2}, i=1,2
$$

Proof: The rate constraint (17) is derived in section III. Then we give the proof of the constraint (18) and (19). Based on the signals (10) received by the CP, the data rate of user $i$ is constrained by

$$
\begin{aligned}
& r_{1 c} \leq I\left(x_{1 c} ; \widehat{Y}_{1} \mid x_{2 p}\right) \\
& r_{2 c} \leq I\left(x_{2 c} ; \widehat{Y}_{2} \mid x_{1 p}, x_{1 c}\right) .
\end{aligned}
$$

Because we assume that the $\mathrm{CP}$ decodes the user data after it receives the decoded data $x_{i p}, i=1,2, x_{i p}$ can be used as side information. Thus the data $x_{i c}$ is decoded without the interference of $x_{\bar{i} p}$. The order of SIC scheme is fixed, so the decoded data $x_{1 c}$ is used as side information when decoding the data $x_{2 c}$. Therefore, the mutual information can be easily calculated.

$$
\begin{aligned}
& I\left(x_{1 c} ; \widehat{Y}_{1} \mid x_{2 p}\right) \\
= & h\left(\widehat{Y}_{1} \mid x_{2 p}\right)-h\left(\widehat{Y}_{1} \mid x_{2 p}, x_{1 c}\right) \\
= & \log _{2}\left(1+\frac{a_{1}^{2} \rho_{1 c} H_{11}^{H} H_{11}}{a_{1}^{2} \rho_{2 c} H_{12}^{H} H_{12}+a_{1}^{2} \sigma^{2}+Q_{1}}\right) \\
& I\left(x_{2 c} ; \widehat{Y}_{2} \mid x_{1 p}, x_{1 c}\right) \\
= & h\left(\widehat{Y}_{2} \mid x_{1 p}, x_{1 c}\right)-h\left(\widehat{Y}_{2} \mid x_{1 p}, x_{1 c}, x_{2 c}\right) \\
= & \log _{2}\left(1+\frac{a_{2}^{2} \rho_{2 c} H_{22}^{H} H_{22}}{a_{2}^{2} \sigma^{2}+Q_{2}}\right) .
\end{aligned}
$$

Based on the analysis above, we can define the achievable rate region, which is presented as below:

Definition 1: The achievable rate region is defined as

$$
\mathcal{R}\left(C_{1}, C_{2}\right)=\bigcup_{\rho_{1 p}, \rho_{1 c}, \rho_{2 p}, \rho_{2 c}}\left(r_{1}, r_{2}\right)
$$

where $r_{i}=r_{i p}+r_{i c}$, and $r_{i p}, r_{i c}$ satisfy the constraint in proposition 1 .

For the fixed backhaul capacity, the achievable rate region is obtained by the convex hull operation on set $\left(r_{1}, r_{2}\right)$. However, it is very complex to operate on the convex hull by ergodic power allocation, although only the boundary of such rate region is particularly interesting for us. As a matter of fact, points on the boundary of rate region are what we need most. In order to reduce the complexity, an optimized method is proposed to obtain such points in next section.

\section{Performance analysis of the proposed decoding scheme}

In this subsection, we analyze the performance of the proposed decoding scheme. For simplicity of analysis, only single-user scenario is considered. The user rate is expressed as:

$$
\begin{aligned}
& r=r_{1 p}+r_{1 c} \\
& =\log _{e}\left(1+\frac{\alpha p_{1}}{\alpha p_{2}+\sigma^{2}}\right)+\log _{e}\left(1+\frac{\alpha p_{2}}{\sigma^{2}+Q_{1}}\right) \\
& =C-\log _{e}\left(1-\frac{\sigma^{2}}{\alpha p_{2}+\sigma^{2}}+\frac{e^{C} \sigma^{2}}{\alpha p_{1}+\alpha p_{2}+\sigma^{2}}\right)
\end{aligned}
$$

where the power $p_{1}, p_{2}$ and the quantization nois are constrained by

$$
\begin{aligned}
& p_{1}+p_{2} \leq P \\
& Q_{1}=\frac{\alpha p_{2}+\sigma^{2}}{e^{C-r_{1 p}}-1} \\
& r_{1 p}=\log _{e}\left(1+\frac{\alpha p_{1}}{\alpha p_{2}+\sigma^{2}}\right) .
\end{aligned}
$$

We want to optimize the maximum rate by optimal power allocation, which is obtained by solving the optimization problem below.

$$
\begin{array}{ll}
\max & C-\log _{e}\left(1-\frac{\sigma^{2}}{\alpha p_{2}+\sigma^{2}}+\frac{e^{C} \sigma^{2}}{\alpha p_{1}+\alpha p_{2}+\sigma^{2}}\right) \\
\text { s.t. } & p_{1}+p_{2} \leq P \\
& p_{1}, p_{2} \geq 0 \\
& r_{1 p} \leq C
\end{array}
$$

The object function is increasing monotonically as $P_{1}$ goes up, however the monotonicity of $p_{2}$ varies with the change of $C, \alpha$ and $\sigma$. The property of object function is observed by the partial derivatives. For lack of space, we only give the partial derivatives results of the variables $p_{1}, p_{2}$, which is shown on the next page header. Thus, for any $\mathrm{C}$, the $\frac{\partial r}{\partial p_{1}} \geq 0$ satisfies. When the $C$ is low, the $\frac{\partial r}{\partial p_{2}} \leq 0$, the optimal rate is obtained by forcing $p_{1}=P, p_{2}=0$. However, when the $C$ is high, there exists a tradeoff of $p_{1}, p_{2}$. The optimal $p_{1}, p_{2}$ can be obtained by solving KKT conditions:

$$
\begin{aligned}
\frac{\partial r}{\partial p_{1}}+\lambda_{1}+\lambda_{2}-v_{1} & =0 \\
\frac{\partial r}{\partial p_{2}}+\lambda_{1}-\left(e^{c}-1\right) \lambda_{2}-v_{2} & =0 \\
\lambda_{1}\left(p_{1}+p_{2}-P\right) & =0 \\
\lambda_{2}\left(p_{1}-\left(e^{C}-1\right)\left(p_{2}+\frac{\sigma^{2}}{\alpha}\right)\right) & =0 \\
v_{1} p_{1} & =0 \\
v_{2} p_{2} & =0
\end{aligned}
$$

We can get the optimal $p_{1}$ and $p_{2}$ easily from the solution of the above simultaneous equations.

\section{Optimized Method by Splitting Total Rate}

From [11], we know that the rate region can be converted to the power region and vice versa. We will optimize the total 


$$
\begin{gathered}
\frac{\partial r}{\partial p_{1}}=\frac{\alpha e^{C} \sigma^{2}\left(\alpha p_{2}+\sigma^{2}\right)}{\left(\alpha p_{1}+\alpha p_{2}+\sigma^{2}\right)\left(\alpha p_{2}\left(\alpha p_{1}+\alpha p_{2}+\sigma^{2}\right)+e^{C} \sigma^{2}\left(\alpha p_{2}+\sigma^{2}\right)\right)} \\
\frac{\partial r}{\partial p_{2}}=\frac{\alpha \sigma^{2}\left(e^{C}\left(\alpha p_{2}+\sigma^{2}\right)^{2}-\left(\alpha p_{1}+\alpha p_{2}+\sigma^{2}\right)^{2}\right)}{\left(\alpha p_{2}+\sigma^{2}\right)\left(\alpha p_{1}+\alpha p_{2}+\sigma^{2}\right)\left(\alpha p_{2}\left(\alpha p_{1}+\alpha p_{2}+\sigma^{2}\right)+e^{C} \sigma^{2}\left(\alpha p_{2}+\sigma^{2}\right)\right)}
\end{gathered}
$$

rate to obtain the boundary of the rate region firstly. Then, we will verify the feasibility of the split rate by optimizing the power allocation.

For a fixed $\alpha \in[0,1]$, the points on the boundary of the rate region can be derived by solving the following problem (P2), which is described as:

$$
\begin{array}{ll}
\max . & R \\
\text { s.t. } & r_{1}=\alpha R \\
& r_{2}=(1-\alpha) R \\
& r_{i}=r_{i p}+r_{i c} \\
& r_{i} \leq C_{i}, i=1,2 \\
& r_{1}+r_{2} \leq C_{1}+C_{2} \\
& r_{i p} \geq 0, r_{i c} \geq 0, i=1,2
\end{array}
$$

All the rate in problem above was defined in definition 1 and the constraints follow (4) and (5). This problem can be solved using a bisection method over $R$. The algorithm is shown as below:

1) $R_{\min }=0, R_{\max }=C_{1}+C_{2}$

2)Repeat until $R_{\max }-R_{\min } \leq \epsilon$

a)Set $R=\left(R_{\min }+R_{\max }\right) / 2$

b)Decide feasibility of $\mathrm{R}$

c)If feasible, $R_{\min }=R$, else $R_{\max }=R$

While the feasibility of $R$ is determined based on the method proposed in [12]. When $\alpha$ is fixed, whether $R$ is feasible or not is determined by the feasibility of the rate pair $\left(r_{1}, r_{2}\right)$.

\section{A. Feasibility of a given rate pair}

Assume sum rate $R$ and $\alpha$ is fixed, establishing feasibility hinges on the following conditions:

- Make sure that $r_{i}$ can not exceed $C_{i}, i=1,2$.

- If given the backhaul constraint, the feasibility of the data rate pair $\left(r_{1}, r_{2}\right)$ is established by solving the following optimized problem (P3):

$$
\begin{array}{ll}
\min . & \sum_{i=1}^{2} \rho_{i p}+\rho_{i c} \\
\text { s.t. } & r_{i p}+r_{i c}=r_{i} \\
& r_{i p} \leq \log _{2}\left(1+\frac{\rho_{i p} H_{i i}^{H} H_{i i}}{\rho_{i c} H_{i i}^{H} H_{i i}+\left(\rho_{\bar{i} p}+\rho_{\overline{i c}}\right) H_{i \bar{i}}^{H} H_{i \bar{i}}+\sigma^{2}}\right) \\
& r_{1 c} \leq \log _{2}\left(1+\frac{a_{1}^{2} \rho_{1 c} H_{11}^{H} H_{11}}{a_{1}^{2} \rho_{2 c} H_{12}^{H} H_{12}+a_{1}^{2} \sigma^{2}+Q_{1}}\right) \\
& r_{2 c} \leq \log _{2}\left(1+\frac{a_{2}^{2} \rho_{2 c} H_{22}^{H} H_{22}}{a_{2}^{2} \sigma^{2}+Q_{2}}\right) \\
& \rho_{i p}+\rho_{i c} \leq P_{i}, i=1,2
\end{array}
$$

It is obvious that if there is a minimum power allocation which satisfies the constraints in P3, the rate pair $\left(r_{1}, r_{2}\right)$ is inside the rate region and feasible. Unfortunately, when no such a power allocation exists here, the rate pair is out of the rate region and infeasible. Therefore, we must reduce the sum rate, which is the main idea of the bisection method.

\section{Numerical Results}

In this section, we simulate the rate region within different cases. The simulation parameters are set as follows:

Each BS and each user have only one antenna. The capacities of two backhaul links are set as $C_{1}=C_{2}=C$. The maximum power of two users is defined as $P_{1}=P_{2}=1$. The power of noise is set as $\sigma^{2}=0.1$. The channel coefficients are set as $H_{i j} \sim \operatorname{eN}\left(0, \sigma_{i j}^{2}\right)$ and fixed as $\sigma_{i i}^{2}=1 ; \sigma_{i \bar{i}}^{2}=0.5, i=$ 1,2 . For different schemes, we label IC for the interference channel, JD for the joint decoding scheme in [7], and the PS for our proposed scheme. As can be seen in Fig.3, the IC and PS

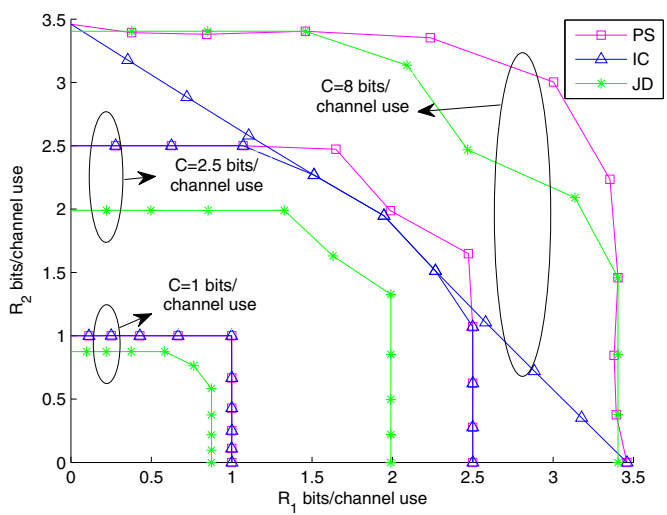

Fig. 3. Rate region with different backhaul capacities for $N_{t}=1, P_{1}=$ $P_{2}=1, \sigma^{2}=0.1, C_{1}=C_{2}=C$.

schemes outperform JD scheme in quite low backhaul capacity (such as 1,2.5 bits/channel use). Under the circumstances, the system is backhaul-limited and the JD scheme is constrained by the higher quantization power. However in higher backhaul capacity ( 8 bits/channel use), the PS scheme is superior to the other two schemes, when the system becomes limited by the achievable rate over the air interface. Since we do not obtain the rate region by time sharing different decoding strategies, the regions are not always as smooth and can be not convex. Fig.4 shows the maximum sum rates achieved by the proposed scheme under the fixed channel. As $\mathrm{C}$ increases, the saturation of the sum rate at $2 \mathrm{C}$ occurs at higher signal-noise-ratio (SNR). Also for quite low $\mathrm{C}$, the maximum sum rate of $2 \mathrm{C}$ is achieved at low SNR. In this figure, we also observe how much of the rates $r_{i p}$ contribute to the total data rate. At low SNR and low 


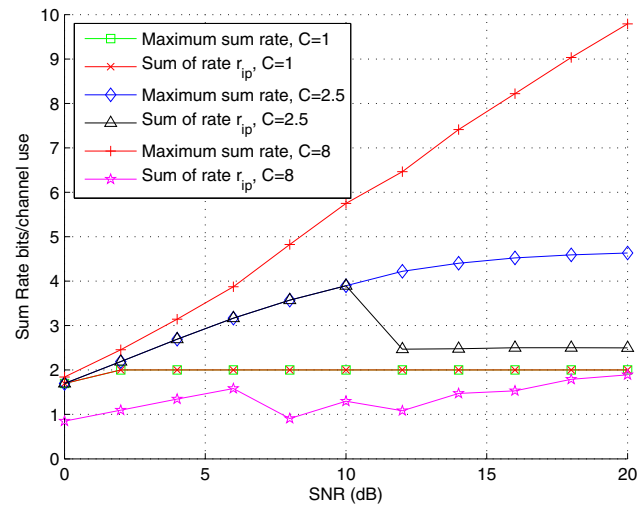

Fig. 4. Maximum sum rate versus SNR for $C=1,2.5$ and 8 bit$\mathrm{s} / \mathrm{channel}$ use. The figure also shows how much of the rates $r_{i p}$ contribute.

$\mathrm{C}$, all the total rate comes from the rate $r_{i p}$. That is because the decoding in the BS can save the backhaul capacity and low SNR can not resist the quantization power if decoding in the CP. However, for high SNR, the rate $r_{i p}$ will reduce and the total rate comes more from the decoding in the CP. Fig.5 shows

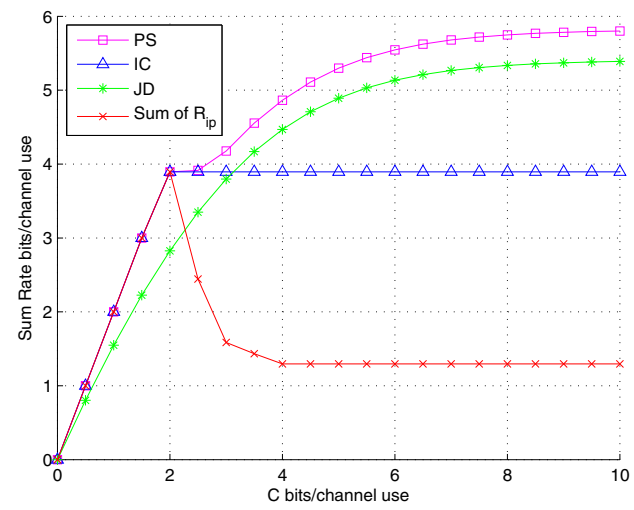

Fig. 5. Maximum sum rate with different backhaul capacity for $N_{t}=$ $1, P_{1}=P_{2}=1, \sigma^{2}=0.1, C_{1}=C_{2}=C$.

the achieved maximum sum rate versus $\mathrm{C}$ for a $10 \mathrm{~dB}$ SNR. For low $\mathrm{C}$, the proposed scheme is the same as the IC scheme, which is also shown in Fig.3. As C increases, the PS scheme outperforms the IC scheme and the JD scheme. The sum rate of $r_{i p}$ will convergence as the increasing $\mathrm{C}$. When the $\mathrm{C}$ is less than 2 bits/channel use, the system is backhaul-limited, and the achievable maximum sum rate is $2 \mathrm{C}$. When the $\mathrm{C}$ is more than 8 bits/channel use, the system is constrained by the SNR. When $\mathrm{C}$ is higher, the quantization power is quite low, thus the air interface is constrained by the achievable maximum power. When $\mathrm{C}$ is more than $4 \mathrm{bits} / \mathrm{channel}$ use, the sum rate of $r_{i p}$ is convergent and the gain of the total rate is from the gain of lower quantization noise. The property can be derived by solving the KKT conditions in subsection III-D.

\section{CONCLUSION}

This paper proposes an optimized data splitting and detection scheme in uplink network MIMO with finite-capacity backhaul links. Each BS has the ability of decoding, and it can decode its supporting users' partial message and compress the left signals to forward to the $\mathrm{CP}$. The $\mathrm{CP}$ decompresses its received quantized signals and jointly decodes the rest message. We derive the constraint of the over-the-air interface and analyze the performance of the partial decoding scheme. The proposed optimization method acquires the boundary of the achievable rate region, and the optimized method reduces the complexity of exhaustive search method. Finally, the simulations verify that the proposed approach outperforms IC and the joint decoding scheme. The results also show that the proposed scheme inherits the advantages of both IC scheme and joint decoding scheme.

\section{ACKNOWLEDGEMENT}

This work has been supported by Industrialization of Commercial Equipments in TD-LTE-A, MIIT of China under grant 2015ZX03001014-002, the National High Technology Research and Development Program of China (863 Program) through grant 2014AA01A702 and the National Natural Science Foundation of China through grant 61171112. The numerical calculations in this paper have been done on the supercomputing system in the Supercomputing Center of University of Science and Technology of China.

\section{REFERENCES}

[1] Kaviani, Saeed, and Witold A. Krzymien. "Multicell scheduling in network MIMO." Global Telecommunications Conference (GLOBECOM 2010), 2010 IEEE. IEEE, 2010.

[2] Gesbert, David, et al. "Multi-cell MIMO cooperative networks: A new look at interference." Selected Areas in Communications, IEEE Journal on 28.9 (2010): 1380-1408.

[3] Venkatesan, Sivarama, Angel Lozano, and Reinaldo Valenzuela. "Network MIMO: Overcoming intercell interference in indoor wireless systems." Signals, Systems and Computers, 2007. ACSSC 2007. Conference Record of the Forty-First Asilomar Conference on. IEEE, 2007.

[4] Grieger, Michael, Patrick Marsch, and Gerhard Fettweis. "Uplink base station cooperation by iterative distributed interference subtraction." Personal, Indoor and Mobile Radio Communications, 2009 IEEE 20th International Symposium on. IEEE, 2009.

[5] Marsch, Patrick, and Gerhard Fettweis. "Uplink CoMP under a constrained backhaul and imperfect channel knowledge." Wireless Communications, IEEE Transactions on 10.6 (2011): 1730-1742.

[6] Sanderovich, Amichai, et al. "Communication via decentralized processing." Information Theory, IEEE Transactions on 54.7 (2008): 3008-3023.

[7] Zhou, Lei, and Wei Yu. "Uplink multicell processing with limited backhaul via successive interference cancellation." Global Communications Conference (GLOBECOM), 2012 IEEE. IEEE, 2012.

[8] Cover T M, Thomas J A. Elements of information theory[M]. John Wiley \& Sons, 2012.

[9] Bussgang J J. "Crosscorrelation functions of amplitude-distorted Gaussian signals." 1952.

[10] Behravan, Ali, and Thomas Eriksson. "Analysis of distortion in a memoryless bandpass nonlinearity." Proc. Nordic Radio Symposium. 2004.

[11] Mohseni, Mehdi, Rui Zhang, and John M. Cioffi. "Optimized transmission for fading multiple-access and broadcast channels with multiple antennas." Selected Areas in Communications, IEEE Journal on 24.8 (2006): 1627-1639.

[12] Zakhour, Randa, and David Gesbert. "On the value of data sharing in constrained-backhaul network MIMO." Proc. International Zurich Seminar on Communications. 2010. 